\title{
Rachelle Chadwick (2018): Bodies That Birth. Vitalizing Birth Politics
}

\author{
Oxon, New York: Routledge. 225 Seiten, \$ 52,95
}

\author{
Marietta Mayrhofer-Deak
}

(C) Der/die Autor(en) 2022

Die Autorin Rachelle Chadwick ist Senior Lecturer für Soziologie an der Universität Pretoria (University of Pretoria) in Südafrika. Sie war NRF Research Career Fellow an der Universität Kapstadt. Ihre Forschungsinteressen kreisen um die Themen reproduktive Politik, feministische Theorien, geburtshilfliche Gewalt, neue Materialismen, experimentelle qualitative (Körper-)Studien.

Ihre erste Monographie „Bodies that Birth“ fußt auf zwei Forschungsprojekten: einerseits auf ihrer Dissertation (PhD), in der sich die Autorin auf Geburtserfahrungen von weißen südafrikanischen Frauen der Mittelschicht konzentrierte, welche eine Hausgeburt planten oder für die Geburt ihres Kindes einen Wunsch-Kaiserschnitt wählten. Das Kernstück der Arbeit bildeten qualitative Interviews mit Frauen vor und nach der Geburt (insgesamt 27, davon 24 face to face und 3 via E-Mail Korrespondenz). In einem Folgeforschungsprojekt beschäftigte sich die Autorin sodann mit den Geburtserfahrungen von 35 Schwarzen südafrikanischen Frauen mit geringem Einkommen, die in informellen Siedlungen in der Western Cape Region lebten. 17 dieser Frauen brachten ihr Kind in einem öffentlichen Spital zur Welt, 16 nahmen den Dienst einer Hebamme in Anspruch. In dieser Gruppe gab es 8 Notkaiserschnitte und keine Wunschkaiserschnitte.

Im Hinblick auf den Forschungsprozess merkt die Autorin an, dass ihre eigene Identität und soziale Herkunft - die Autorin ist selbst (noch) kinderlos und gehört der gebildeten südafrikanischen weißen Mittelschicht an - den Feldzugang im ersten Projekt erleichterte, im zweiten Projekt jedoch erschwerte. Einerseits war die Zusammenarbeit mit einer lokalen NGO notwendig, um überhaupt Interviewpartnerinnen zu finden, andererseits war eine Begleitung durch Sozialarbeiter*innen in den Wohngebieten notwendig, um die Autorin vor Gefahren zu schützen. Zu-

Marietta Mayrhofer-Deak ( $\square)$

Institut für Soziologie, Universität Wien, Rooseveltplatz 2, 1090 Wien, Österreich

E-Mail: marietta.mayrhofer-deak@univie.ac.at 
dem kam es zu sprachlichen Schwierigkeiten. Die Autorin führte 7 Interviews mit muttersprachlichen isiXhosa Sprecherinnen - isiXhosa ist die am weitesten verbreitete afrikanischen Sprache in Südafrika - in Englisch und 28 Interviews mit muttersprachlichen Afrikaans Sprecherinnen in Afrikaans - wobei die Autorin diese Sprache nicht perfekt beherrscht.

Trotz der genannten Schwierigkeiten und den daraus resultierenden Beschränkungen versucht die Autorin in „Bodies that Birth“ ein vielschichtiges Bild von den Geburtserfahrungen südafrikanischer Frauen unterschiedlicher sozialer Herkunft zu skizzieren und die sozialen Bedingungen, welche diese Erfahrungen framen, in den Mittelpunkt der Betrachtung zu rücken: Körperpolitik ebenso wie soziale Ungleichheiten entlang der Achsen race, class und sozio-materielle Positionen (S. 6-7). Ihr Ziel ist es, Frauen, die über Geburt erzählen, als ambige, intersektionale körperliche Subjekte zu repräsentieren, die in einem zähen Netz von Machtbeziehungen gefangen sind und sich zwischen Akten des Widerstands, des offenen Einverständnisses, der Passivität und des geheimen Einverständnisses bewegen (S. 7). Nicht berücksichtigt werden die unterschiedlichen philosophisch und religiös geprägten Mythen und kulturellen Interpretationen des Geburtsprozesses (Forschungen in diesem Sinn unternahm z.B. die Anthropologin Alma Gottlieb vgl. „The Afterlife is where we come from. The Culture of Infancy in West Africa“, 2004). Anzumerken ist, dass die Autorin auf kulturelle Besonderheiten im südafrikanischen Kontext - auch nicht im allgemeinsten Sinn - en detail eingeht, was gerade für die internationale Leserschaft aber interessant gewesen wäre. Ein gewisses Manko stellt auch die völlige Außerachtlassung quantitativer Daten dar.

Das Denken der Autorin ist von europäischen und US-amerikanischen poststrukturalistischen Intellektuellen, u. a. von Michel Foucault, Gilles Deleuze, Félix Guattari, Donna Haraway und Julia Kristeva beeinflusst. „Southern Theories“ (Raewyn Connell) stellen keine zentralen Bezugspunkte dar. Diese Absenz von Theorien und insbesondere Theoretikerinnen aus dem globalen Süden ist im Hinblick auf die Themenwahl der Autorin bedauernswert und jedenfalls mit einer Inkongruenz verbunden, denn: in Anlehnung an Gilles Deleuze geht es der Autorin just um ein Aufbrechen von Strukturen und die Visualisierung von Machtbeziehungen; mit Julia Kristeva versucht sie, die Beziehung zwischen Körper und Sprache in ihrer Vielfalt zu thematisieren. Eine Stimme beinhalte zugleich eine Vielstimmigkeit, die es wahrzunehmen gelte, argumentiert sie unter Bezugnahme auf dialogische Ansätze der Narrationsanalyse (u. a. Arthur W. Frank, S. 19).

Schlüssig scheint hingegen die bewusste Ablehnung von qualitativen Analysemethoden, die auf einer Komplexitätsreduktion mittels Kategorienbildung fußen. Würden solche nicht gerade ,patterns of difference that make a difference“ (S. 14) Diskontinuitäten, Leerstellen, die Mehrstimmigkeit und die hermeneutischen Unterbrechungen in den Erzählungen unsichtbar machen (S. 19)? Kurz: unsichtbar machen, wie (Ohn-)Macht erfahren wird und wie sich das Soziale in körperliche Empfindungen einschreibt?

Die Autorin entscheidet sich für eine assemblage, die auch im Aufbau des Buches zum Ausdruck kommt. Nach der Einleitung folgt ein Kapitel über die politischen Rahmenbedingungen und Diskurse, in welche Geburten eingebettet sind. Die Autorin geht u. a. auf biomedizinische und psychologische Aspekte (die dominante euro- 
amerikanische Erzählung vom gebärenden Körper als defekte Maschine, S. 28), aber auch auf das koloniale Erbe in Südafrika ein, das sie insbesondere in fortwährenden Diskriminierungen und Mythen über ,rassifizierte“ Körper sieht (S. 29). Um sichtbar zu machen, wie sich diese Rahmenbedingungen in die Erzählungen und Körperempfindungen der Frauen einschreiben, wendet sie eine besonders detailgetreue Transkriptionsmethode an, welche Lautmalereien und körperbezogene Ausrufe/Laute besonders hervorhebt. In den folgenden Kapiteln - 3. Clockwork bodies, 4. Risky bodies, 5. Violated Bodies, 6. Resistant Bodies - fügt sie immer wieder Auszüge aus den geführten Interviews in den Text - der primär Narrationsanalyse ist - ein.

Im Hinblick auf die theoretische Ausrichtung und den Umgang mit dem empirischen Material besonders beachtlich ist das fünfte Kapitel. In diesem beschäftigt sich die Autorin mit dem Thema „Gewalt unter der Geburt“ und rückt referierend auf Johan Galtung, Pierre Bourdieu, Slavoj Žižek und Claudio Colaguori „normalisierte“ und ,,versteckte“ Gewaltformen eindrucksvoll in den Mittelpunkt der Betrachtung.

Die „,kollektive Assemblage von Stimmen“ dient der Autorin als Ausgangspunkt, um darüber nachzudenken, was generell zum emotionalen Wohlbefinden der Frauen im Hinblick auf die Geburt beiträgt und wie Geburtspolitiken vitalisiert werden könnten (S. 171). Worauf kommt es an?

Die Autorin resümiert: Nicht die Art der Geburt ist entscheidend, nicht primär medizinische und technische Aspekte, sondern die affektiven Energien, flows und Beziehungen, die in einem spezifischen Geburtssetting zirkulieren (S. 193) sind es. „Gute“ Geburten werden besonders oft mit dem Gefühl der „Wärme“ in Verbindung gebracht, eine Wärme, die in einem unterstützenden Umfeld entsteht (S. 194), während ,schlechte“ Geburten oft von dem Gefühl des Unsichtbarwerdens begleitet werden und mit einem Mangel an ,embodied care“ (Maurice Hamington) in enger Verbindung stehen. Entscheidend sind soziale Aspekte: neben Freundlichkeit, Wärme und Fürsorge insbesondere erlebte Sicherheit (sowohl ontologisch wie auch physisch) und die Achtung der Würde der gebärenden Frau. Auf beeindruckende Weise schildert die Autorin, wie sehr es in diesem Zusammenhang auch auf einen diskriminierungsfreien Umgang ankommt - sei es im Hinblick auf race, sei es im Hinblick auf sozioökonomische Ungleichheiten (S. 196ff.). Die Geburtspolitik zu vitalisieren bedeute, genau diese Aspekte ins Zentrum der Betrachtung und der gesundheitspolitischen Strukturen zu rücken (S. 199).

Fazit: Die Autorin zeigt mit ihrer Forschung, trotz der genannten Schwierigkeiten, zahlreiche bisher nur unzureichend belichtete soziologische Aspekte rund um das Thema Geburt auf und weist mit ihrer Arbeit auf weiteren Forschungsbedarf hin - etwa hinsichtlich der verbindenden Betrachtung von politischer Ökonomie und feministischen/menschenrechtlichen Anliegen.

Funding Open access funding provided by University of Vienna.

Open Access Dieser Artikel wird unter der Creative Commons Namensnennung 4.0 International Lizenz veröffentlicht, welche die Nutzung, Vervielfältigung, Bearbeitung, Verbreitung und Wiedergabe in jeglichem Medium und Format erlaubt, sofern Sie den/die ursprünglichen Autor(en) und die Quelle ordnungsgemäß nennen, einen Link zur Creative Commons Lizenz beifügen und angeben, ob Änderungen vorgenommen wurden. 
Die in diesem Artikel enthaltenen Bilder und sonstiges Drittmaterial unterliegen ebenfalls der genannten Creative Commons Lizenz, sofern sich aus der Abbildungslegende nichts anderes ergibt. Sofern das betreffende Material nicht unter der genannten Creative Commons Lizenz steht und die betreffende Handlung nicht nach gesetzlichen Vorschriften erlaubt ist, ist für die oben aufgeführten Weiterverwendungen des Materials die Einwilligung des jeweiligen Rechteinhabers einzuholen.

Weitere Details zur Lizenz entnehmen Sie bitte der Lizenzinformation auf http://creativecommons.org/ licenses/by/4.0/deed.de.

Dr. Marietta Mayrhofer-Deak Lektorin am Institut für Soziologie der Universität Wien. Schwerpunkte in Lehre und Forschung: Soziologische Theorien, Geschichte der Soziologie, Perspektiven aus dem globalen Süden. 